\title{
ZIV-ZAKAI LOWER BOUND FOR UWB BASED TOA ESTIMATION WITH UNKNOWN INTERFERENCE
}

\author{
Adrià Gusi Amigó $o^{\dagger}$ Pau Closas ${ }^{\ddagger}$, Achraf Mallat ${ }^{\dagger}$ and Luc Vandendorpe ${ }^{\dagger}$ \\ ${ }^{\dagger}$ ICTEAM Institute, Louvain School of Engineering, Université catholique de Louvain \\ place du Levant 2, B-1348 Louvain-la-Neuve, Belgium \\ ${ }^{\ddagger}$ Centre Tecnològic de Telecomunicacions de Catalunya (CTTC) \\ Av. Carl Friedrich Gauss 7, 08860 Castelldefels, Barcelona (Spain) \\ e-mail: \{adria.gusiamigo,achraf.mallat,luc.vandendorpe\}@uclouvain.be,pclosas@cttc.cat
}

\begin{abstract}
This paper derives the Ziv-Zakai lower bound (ZZLB) for the time of arrival (TOA) estimation in the presence of one interfering pulse from which no a priori knowledge is available. The bound is obtained by including the interference in the system model but only the transmitted pulse as a candidate for the likelihood ratio (LR) test. A compact ZZLB expression that depends on the time delay and amplitude of the interference is obtained. We compare the performance of the first path maximum likelihood estimation (MLE) with the bound as a function of the relative distance between the first path and the interfering path.
\end{abstract}

Index Terms - Time of arrival estimation, Ultra wideband communication, Interference, Ziv-Zakai lower bound

\section{INTRODUCTION}

Ultra-wideband (UWB) signals can provide very accurate positioning thanks to their short duration pulses $[1,2]$. In most of the situations, the estimation of first path time of arrival (TOA) is corrupted by the presence of interference. The interference, such as multipath (MP) interference or multiuser interference (MUI), can modify the shape of the first path and hence, the estimation performance changes. Moreover, the interference is sometimes stronger than the first path, and then large estimation errors take place.

Some authors address the TOA estimation bounds in UWB channels. In [3] the Cramèr-Rao lower bound (CRLB) is given for the joint estimation of MP channel parameters . In [4] the CRLB is given when up to three overlapping MP components are considered. However, the joint estimation of all parameters is very complex and becomes impractical. Moreover, the CRLB is only accurate at high signal to noise ratio (SNR) or for long observation times [5].

Several papers investigate the Ziv-Zakai lower bound (ZZLB) also for UWB channels. The ZZLB is a Bayesian bound well known for taking into account the $a$ priori distribution of the parameter and correctly predicting the mean square error (MSE) behavior over the whole range of SNR $[6,7]$. The ZZLB is obtained by transforming the estimation problem into a binary hypothesis testing problem

The authors would like to thank the FRIA and the FNRS for the financial support, BELSPO for the support of the IAP BESTCOM network, the Spanish Ministry of Economy and Competitiveness project TEC2012-39143 (SOSRAD) and the European Commission in the Network of Excellence in Wireless COMmunications NEWCOM $\sharp$ (contract n. 318306). with an associated probability of error. In [1, 8], ZZLBs for two different cases are considered. First, a perfect measurement bound (PMB) where the nuisance parameters are considered known nonrandom variables, and second, a bound where the nuisance is a set of random variables with known a priori distributions. Both bounds are implemented for realistic UWB multipath channels by averaging over a given number of channel responses (CR). In [9] the ZZLB is derived for convolutive random channels with instantaneous channel knowledge at the receiver, that is to say that the channel realization is perfectly known at the receiver, while in [10] the topic is readdressed considering that the receiver knows the a priori channel distribution. Both papers model the channel as a taped delay line. In [11], ZZLB is derived for MUI. The bound is obtained by handling a probability of error averaged over the MUI distribution.

This paper investigates MSE lower bounds on the TOA in the presence of one interfering pulse when no $a$ priori knowledge about the nuisance parameters governing the interference is available. In the derivation of the bound we consider no channel knowledge at the receiver. The transmitted signal is known to the receiver and its TOA has a uniform prior distribution. Under this conditions, the loglikelihood ratio (LLR) is computed in a situation where the received signal contains interference but only the transmitted signal is a candidate for the associated hypothesis test. This differs from previous ZZLB work, where all the multipaths are considered as a candidate. A compact ZZLB expression that depends on the time delay and amplitude of the interference is obtained. This bound enables to express the MSE as a function of the relative delay between the first path and the interfering path, and of the amplitude ratio between both paths.

The bound is derived for a generic interfering pulse. If the adequate pulse shape, relative delay, and amplitude ratio are employed, more specific scenarios can be analyzed such as multipath propagation channels or multiuser channels.

The paper is organized as follows. In Section 2, we describe the system model. In Section 3, we review the ZZLB. In Section 4 , we derive the probability of error for the system model described in Section 2 and derive the associate ZZLB. In Section 5 numerical results are reported and discussed. Finally, Section 6 concludes the paper.

\section{THE SYSTEM MODEL}

The UWB transmitted signal is $\sqrt{E_{t}} p(t)$, where $p(t)$ is the Gaussian pulse, normalized to satisfy $\int_{-\infty}^{+\infty} p^{2}(t) d t=1$, so that $E_{t}$ is the 
transmitted signal energy. The received signal is given by

$$
\begin{aligned}
r(t) & =s(t-\tau)+s_{i}\left(t-\tau-\tau_{i}\right)+n(t) \\
& =\sqrt{E_{t}} \alpha p(t-\tau)+\sqrt{E_{t}} \alpha_{i} p_{i}\left(t-\tau-\tau_{i}\right)+n(t)
\end{aligned}
$$

where $s(t)$ is the the received useful signal with amplitude $\alpha$ and TOA $\tau, s_{i}(t)$ is the interference signal, $p_{i}(t)$ is the interfering pulse, $\tau_{i}$ and $\alpha_{i}$ are the time delay and amplitude of the interfering pulse, and $n(t)$ is additive white Gaussian noise (AWGN) with double sided spectral density $N_{0} / 2$. The interfering pulse $p_{i}(t)$, whose shape can be different from $p(t)$, is normalized to satisfy $\int_{-\infty}^{+\infty} p_{i}^{2}(t) d t=1$. The time of arrival (TOA) $\tau$ is uniformly distributed on $[0, T]$. For the particular situation where $p_{i}(t)=p(t)$, we encounter the multipath case.

\section{ZIV ZAKAI LOWER BOUND}

In this section we present a short review of the ZZLB. ZZLB provides a bound on the MSE over the a priori probability density function (pdf). The full derivation of the bound can be found in $[6,7]$. We are interested in a lower bound for the mean square estimation error of the time delay

$$
\mathbb{E}\left\{\epsilon^{2}\right\}=\mathbb{E}\left\{(\hat{\tau}-\tau)^{2}\right\}
$$

where $E\{\cdot\}$ denotes the expected value. The ZZLB can be obtained from the identity

$$
\mathbb{E}\left\{\epsilon^{2}\right\}=\frac{1}{2} \int_{0}^{\infty} \operatorname{Pr}\left(|\epsilon| \geq \frac{h}{2}\right) h d h
$$

and lower bounding $\operatorname{Pr}\left(|\epsilon| \geq \frac{h}{2}\right)$. The expression $\operatorname{Pr}\left(|\epsilon| \geq \frac{h}{2}\right)$ is related to a binary detection scheme with equally probable hypotheses

$$
\begin{array}{ll}
H_{1}: \tau=a ; & r(t)=s(t-\tau)+n(t) \mid \tau=a \\
H_{2}: \tau=a+h ; & r(t)=s(t-\tau)+n(t) \mid \tau=a+h
\end{array}
$$

when considering a suboptimal decision scheme where the parameter is first estimated and a nearest-neighbor decision is made afterward

$$
\hat{H}= \begin{cases}H_{1}, & \text { if } \hat{\tau} \leq a+\frac{h}{2} \\ H_{2}, & \text { if } \hat{\tau}>a+\frac{h}{2} .\end{cases}
$$

The probability of error for this suboptimum detector can be lower bounded by the minimum error probability $P_{e}(a, a+h)$ given by the likelihood ratio (LR) test

$$
\Lambda=\frac{p(r(t) \mid a)}{p(r(t) \mid a+h)} \underset{H_{2}}{\stackrel{H_{1}}{\gtrless}} 1 .
$$

The term $\operatorname{Pr}\left(|\epsilon| \geq \frac{h}{2}\right)$ can be shown [7] to be greater or equal to

$$
\int_{-\infty}^{\infty}\left(p_{\tau}(a)+p_{\tau}(a+h)\right) P_{e}(a, a+h) d a,
$$

where $p_{\tau}(\tau)$ is the pdf of the TOA. Given that $p_{\tau}(\tau)$ follows a uniform distribution in the interval $[0, T]$, the lower bound on the estimation error can then be expressed as

$$
\mathbb{E}\left\{\epsilon^{2}\right\} \geq \text { ZZLB }=\frac{1}{T} \int_{0}^{T} h \int_{0}^{T-h} P_{e}(a, a+h) d a d h .
$$

Moreover, when $P_{e}(a, a+h)$ is independent of $a$ we can write $P_{e}(h)$ instead. Assuming this, the ZZLB is given by

$$
\mathrm{ZZLB}=\frac{1}{T} \int_{0}^{T} h(T-h) P_{e}(h) d h .
$$

\section{EVALUATION OF THE ZZLB}

The minimum error probability $P_{e}(a, a+h)$ is given by the LR test from (6). The log-likelihood ratio (LLR) can be obtained upon taking the logarithm

$$
\ln \Lambda=\ln p(r(t) \mid a)-\ln p(r(t) \mid a+h)=\Delta(a)-\Delta(a+h)=\underset{H_{2}}{\stackrel{H_{1}}{\gtrless}} 1
$$

where $\Delta(x)$ is the log-likelihood function of $x$ with the constant terms removed, and it can be written as

$$
\Delta(x)=-\frac{1}{N_{0}} \int_{-\infty}^{+\infty}(r(t)-s(t-x))^{2} d t
$$

where $r(t)$ is the signal corrupted with the interference, as introduced in (1). The minimum error probability is then

$$
\begin{aligned}
P_{e}(a, a+h)= & \operatorname{Pr}\left(\ln \Lambda<0 \mid H_{1}\right) \operatorname{Pr}\left(H_{1}\right) \\
& +\operatorname{Pr}\left(\ln \Lambda>0 \mid H_{2}\right) \operatorname{Pr}\left(H_{2}\right) \\
= & \frac{1}{2} \operatorname{Pr}\left(\ln \Lambda<0 \mid H_{1}\right)+\frac{1}{2} \operatorname{Pr}\left(\ln \Lambda>0 \mid H_{2}\right),
\end{aligned}
$$

where equally likely hypotheses are assumed for the second equality. The remaining probabilities can be obtained as

$$
\begin{aligned}
& \operatorname{Pr}\left(\ln \Lambda<0 \mid H_{1}\right)=\operatorname{Pr}(\Delta(a)-\Delta(a+h)<0 \mid \tau=a) \\
& \operatorname{Pr}\left(\ln \Lambda>0 \mid H_{2}\right)=\operatorname{Pr}(\Delta(a)-\Delta(a+h)>0 \mid \tau=a+h)
\end{aligned}
$$

The log-likelihood function of $a$ is then given by

$$
\begin{aligned}
\Delta(a) & =-\frac{1}{N_{0}} \int_{-\infty}^{+\infty}(r(t)-s(t-a))^{2} d t \\
& =-\frac{1}{N_{0}}\left(E_{r}+E_{s}-2 R_{s r}(a)\right),
\end{aligned}
$$

where $E_{r}=\int_{-\infty}^{+\infty} r(t)^{2} d t$ and $E_{s}=\int_{-\infty}^{+\infty} s(t)^{2} d t=E_{t} \alpha^{2}$ are the energies of $r(t)$ and $s(t)$ respectively, and

$$
\begin{aligned}
R_{s r}(a) & =\int_{-\infty}^{+\infty} s(t-a) r(t) d t \\
& =\int_{-\infty}^{+\infty} s(t-a)\left(s(t-\tau)+s_{i}\left(t-\tau-\tau_{i}\right)+n(t)\right) d t \\
& =R_{s}(-\tau+a)+R_{s s_{i}}\left(-\tau_{i}-\tau+a\right)+n_{0}
\end{aligned}
$$

where

$$
\begin{aligned}
R_{s}(x) & =\int_{-\infty}^{+\infty} \sqrt{E_{t}} \alpha p(t-x) \sqrt{E_{t}} \alpha p(t) d t \\
& =E_{t} \alpha^{2} R_{p}(x)
\end{aligned}
$$

$$
\begin{aligned}
R_{s s_{i}}(x) & =\int_{-\infty}^{+\infty} \sqrt{E_{t}} \alpha p(t-x) \sqrt{E_{t}} \alpha_{i} p_{i}(t) d t \\
& =E_{t} \alpha \alpha_{i} R_{p p_{i}}(x)
\end{aligned}
$$

and $n_{0}=\int_{-\infty}^{+\infty} s(t-a) n(t) d t . \quad R_{p}(x)=\int_{-\infty}^{+\infty} p(t-x) p(t) d t$ is the autocorrelation of $p(t)$ and $R_{p p_{i}}(x)=\int_{-\infty}^{+\infty} p(t-x) p_{i}(t) d t$ is the cross-correlation between the transmitted pulse $p(t)$ and the interference $p_{i}(t)$.

The log-likelihood function of $a+h$ is as follows:

$$
\begin{aligned}
\Delta(a+h) & =-\frac{1}{N_{0}} \int_{-\infty}^{+\infty}(r(t)-s(t-a-h))^{2} d t \\
& =-\frac{1}{N_{0}}\left(E_{r}+E_{s}-2 R_{s r}(a+h)\right)
\end{aligned}
$$


where

$$
\begin{aligned}
R_{s r}(a+h) & =\int_{-\infty}^{+\infty} s(t-a-h) r(t) d t \\
& =R_{s}(-\tau+a+h)+R_{s s_{i}}\left(-\tau_{i}-\tau+a+h\right)+n_{1}
\end{aligned}
$$

where $n_{1}=\int_{-\infty}^{+\infty} s(t-a-h) n(t) d t$. With (16) and (19) the probability in (13) yields

$$
\begin{aligned}
\operatorname{Pr}\left(\ln \Lambda<0 \mid H_{1}\right) & =\operatorname{Pr}\left(R_{s r}(a)-R_{s r}(a+h)<0 \mid \tau=a\right) \\
& =\operatorname{Pr}(S(h)+n<0))
\end{aligned}
$$

where

$$
S(h)=R_{s}(0)-R_{s}(h)+R_{s s_{i}}\left(-\tau_{i}\right)-R_{s s_{i}}\left(h-\tau_{i}\right)
$$

and $n=n_{0}-n_{1}$. In the same way, the probability in (14) leads to

$$
\begin{aligned}
\operatorname{Pr}\left(\ln \Lambda>0 \mid H_{2}\right) & =\operatorname{Pr}\left(R_{s r}(a)-R_{s r}(a+h)>0 \mid \tau=a+h\right) \\
& =\operatorname{Pr}(S(-h)-n<0)) .
\end{aligned}
$$

The probability $\operatorname{Pr}\left(\ln \Lambda<0 \mid H_{1}\right)$ is readily given by

$$
\operatorname{Pr}\left(\ln \Lambda<0 \mid H_{1}\right)=Q\left(\frac{S(h)}{\sigma_{n}}\right)
$$

where $\left.Q(x)=(1 / \sqrt{2 \pi}) \int_{x}^{\infty} \exp \left(-t^{2} / 2\right)\right) d t$ is the Q-function, expressed in terms of the complementary error function as $Q(x)=$ $(1 / 2) \operatorname{erfc}(x / \sqrt{2})$. The noise variance $\sigma_{n}^{2}$ is equal to

$$
\begin{aligned}
\sigma_{n}^{2} & =E\left\{\left(n_{0}-n_{1}\right)^{2}\right\}=E\left\{n_{0}^{2}\right\}+E\left\{n_{1}^{2}\right\}-2 E\left\{n_{0} n_{1}\right\} \\
& =\frac{N_{0}}{2} E_{s}+\frac{N_{0}}{2} E_{s}-N_{0} R_{s}(h)=N_{0}\left(E_{s}-R_{s}(h)\right) .
\end{aligned}
$$

The same can be done for $\operatorname{Pr}\left(\ln \Lambda>0 \mid H_{2}\right)$ and leads to

$$
\operatorname{Pr}\left(\ln \Lambda>0 \mid H_{2}\right)=Q\left(\frac{S(-h)}{\sigma_{n}}\right) .
$$

Note that none of the components from $P_{e}(a, a+h)$ depend on $a$, so we can express the minimum probability of error as

$$
P_{e}(h)=\frac{1}{2}\left(Q\left(\frac{S(h)}{\sigma_{n}}\right)+Q\left(\frac{S(-h)}{\sigma_{n}}\right)\right) .
$$

The ZZLB is readily obtained from (9) by inserting the expression of $P_{e}(h)$ given in (27)

$$
\begin{aligned}
\operatorname{ZZLB}= & \frac{1}{T} \int_{0}^{T} h(T-h) \frac{1}{2} Q\left(\frac{S(h)}{\sigma_{n}}\right) d h \\
& +\frac{1}{T} \int_{0}^{T} h(T-h) \frac{1}{2} Q\left(\frac{S(-h)}{\sigma_{n}}\right) d h .
\end{aligned}
$$

With a change of variable, $h=-m$, the second terms becomes

$$
\begin{aligned}
& \frac{1}{T} \int_{0}^{-T} m(T+m) \frac{1}{2} Q\left(\frac{S(m)}{\sigma_{n}}\right) d m \\
& =\frac{1}{T} \int_{-T}^{0}|m|(T-|m|) \frac{1}{2} Q\left(\frac{S(m)}{\sigma_{n}}\right) d m .
\end{aligned}
$$

Both terms can be unified in a single integral and the final expression of the bound is obtained

$$
\operatorname{ZZLB}=\frac{1}{2 T} \int_{-T}^{T}|h|(T-|h|) Q\left(\frac{S(h)}{\sigma_{n}}\right) d h .
$$

The content of the Q-function can be further expanded as

$$
\begin{aligned}
\frac{S(h)}{\sigma_{n}} & =\frac{E_{s}-R_{s}(h)+R_{s s_{i}}\left(-\tau_{i}\right)-R_{s s_{i}}\left(h-\tau_{i}\right)}{\sqrt{N_{0}\left(E_{s}-R_{s}(h)\right)}} \\
& =\alpha^{2} E_{t} \frac{1-R_{p}(h)+\frac{\alpha_{i}}{\alpha}\left(R_{p p_{i}}\left(-\tau_{i}\right)-R_{p p_{i}}\left(h-\tau_{i}\right)\right)}{\sqrt{N_{0} \alpha^{2} E_{t}\left(1-R_{p}(h)\right)}} \\
& =\sqrt{\operatorname{SNR}} \frac{1-R_{p}(h)+\frac{\alpha_{i}}{\alpha}\left(R_{p p_{i}}\left(-\tau_{i}\right)-R_{p p_{i}}\left(h-\tau_{i}\right)\right)}{\sqrt{1-R_{p}(h)}}
\end{aligned}
$$

where SNR $=\frac{\alpha^{2} E_{t}}{N_{0}}$ is the received signal to noise ratio.

We can evaluate the bound when no interference is present. For $\alpha_{i}=0$ the probability of error is as follows

$$
P_{e}(h)=Q\left(\sqrt{\mathrm{SNR}} \frac{1-R_{p}(h)}{\sqrt{1-R_{p}(h)}}\right)=Q\left(\sqrt{\operatorname{SNR}\left(1-R_{p}(h)\right)}\right)
$$

and the bound is equal to

$$
\begin{aligned}
\left.\operatorname{ZZLB}\right|_{\alpha_{i}=0} & =\frac{1}{2 T} \int_{-T}^{T}|h|(T-|h|) Q\left(\sqrt{\operatorname{SNR}\left(1-R_{p}(h)\right)}\right) d h \\
& =\frac{1}{T} \int_{0}^{T} h(T-h) Q\left(\sqrt{\operatorname{SNR}\left(1-R_{p}(h)\right)}\right) d h(33)
\end{aligned}
$$

where the symmetry of $R_{p}(h)$ is applied. The bound matches with the expression given in [6].

Another analysis can be made from (30). It can be shown that when $R_{p p_{i}}(x)$ is symmetric the same MSE is obtained for a delay $\tau_{i}=\theta_{i}$ and $\tau_{i}=-\theta_{i}$. By a change of variable, $h=-m$, the expression (30) yields to

$$
\text { ZZLB }=\frac{1}{2 T} \int_{-T}^{T}|m|(T-|m|) Q\left(\frac{S(-m)}{\sigma_{n}}\right) d m .
$$

Therefore, the term $S(h)$ delivers the same bound as $S(-h)$. Observing $S(h)$ in (22), this is equivalent to mentioning that $R_{s s_{i}}(h-$ $\left.\tau_{i}\right)$ generates the same bound as $R_{s s_{i}}\left(-h-\tau_{i}\right)$. Assuming that $R_{p p_{i}}(x)$ is symmetric, we can write $R_{s s_{i}}\left(-h-\tau_{i}\right)=R_{s s_{i}}\left(h+\tau_{i}\right)$. It can be then noticed that $R_{s s_{i}}\left(h-\tau_{i}\right)$ and $R_{s s_{i}}\left(h+\tau_{i}\right)$ derive the same MSE values. Since the only difference between the terms is the sign preceding $\tau_{i}$, we can write that

$$
\left.\mathrm{ZZLB}\right|_{\tau_{i}=\theta_{i}}=\left.\mathrm{ZZLB}\right|_{\tau_{i}=-\theta_{i}}
$$

when $R_{p p_{i}}(x)$ is symmetric. Note that when the interfering pulse $p_{i}(t)$ is equal to $p(t)$ the cross-correlation $R_{s s_{i}}(x)$ becomes equal to the autocorrelation of $p(t)$ and hence, it is always symmetric.

\section{RESULTS AND DISCUSSION}

In this section we present numerical and simulation results. For both the pulse $p(t)$ and the interfering pulse $p_{i}(t)$ the second derivative of a Gaussian (doublet) is employed

$$
p(t)=p_{i}(t)=\frac{1}{\sqrt{3 T_{m} / 8}}\left(1-4 \pi\left(\frac{t}{T_{m}}\right)^{2}\right) e^{-2 \pi\left(\frac{t}{T_{m}}\right)^{2}}
$$

where the pulse has been normalized and $T_{m}$ is a variable that affects the width of the pulse, which is set to $1 \mathrm{~ns}$. The auto-correlation of the doublet is

$$
R_{p}(x)=\left(1-4 \pi\left(\frac{x}{T_{m}}\right)^{2}+\frac{4 \pi^{2}}{3}\left(\frac{x}{T_{m}}\right)^{4}\right) e^{-\pi\left(\frac{x}{T_{m}}\right)^{2}} .
$$


The bound is compared with the performance of the first path maximum likelihood estimation (MLE). The estimator is as follows

$$
\hat{\tau}=\arg \max _{\tau} \int_{-\infty}^{\infty} r(t) s(t-\tau) d t .
$$

Figure 1 reports the root mean square error (RMSE) as a function of the interference delay $\tau_{i}$. The ZZLB and the MLE are shown for amplitude ratios $\frac{\alpha_{i}}{\alpha}=0.5$ and $\frac{\alpha_{i}}{\alpha}=-0.5$, with delay $\tau_{i}$ ranging from 0 to $2.5 \mathrm{~ns}$. The ZZLB when no interference is present $\left(\frac{\alpha_{i}}{\alpha}=0\right)$ is also shown as a reference. The SNR has been fixed to $30 \mathrm{~dB}$. From the figure it can be noticed that the MLE performs close to the bound for both cases. It can be observed that the RMSE varies noticeably as the delay $\tau_{i}$ changes. Two main behaviors can be seen, on the one hand situations where the RMSEs are close to the reference bound (no interference) and on the other hand RMSEs that are many times higher than the reference bound.

The first case is observable for values of $\tau_{i}$ around 0 and $0.5 \mathrm{~ns}$. For these time delays, a collision with the main or second lobe of the interfering pulse occurs. Hence, the shape of the pulse is slightly modified and only the received energy changes. When the interfering lobe has positive amplitude, a positive collision is taking place and RMSEs below the reference bound are obtained. For negative amplitude, the received energy decreases and worse RMSEs are obtained, above the reference curve. For instance, at $0 \mathrm{~ns}$ the interfering pulse with positive amplitude is generating a positive collision and better RMSEs are obtained, whereas the negative pulse provoques a destructive collision and worse estimates are observed. At $0.5 \mathrm{~ns}$ the reversed situation takes place since the pulse of interest is affected by the secondary lobe of the interfering pulse, which is negative for $\frac{\alpha_{i}}{\alpha}=0.5$ and positive for $\frac{\alpha_{i}}{\alpha}=-0.5$.

The second case takes place for values of $\tau_{i}$ around 0.25 and $0.75 \mathrm{~ns}$. To better understand this behavior, in Figure 2 the received signal is shown when the interfering pulse parameters are $\frac{\alpha_{i}}{\alpha}=0.5$ and $\tau_{i}=0.3 \mathrm{~ns}$. From the figure it appears that the collision of the first path and the interference generates a wider received pulse shifted to the right around $0.1 \mathrm{~ns}$. More accurately, in Figure 1 it can be seen that the RMSE is equal to $0.08 \mathrm{~ns}$, value consistent with Figure 2. The collision of separeted pulses generates biased MLE estimates which are correctly bounded by the ZZLB.

\section{CONCLUSIONS AND FUTURE WORK}

This paper has derived a ZZLB for the TOA estimation in the presence of one interfering pulse from which no a priori knowledge is available. The bound has been obtained by including the interference in the system model but only the transmitted pulse as a candidate for the LR test. A compact ZZLB expression that depends on the time delay and amplitude of the interference has been obtained. The bound has been illustrated as a function of the relative distance between the first path and the interfering path and compared with the performance of the MLE.

The bound is a unique framework to analyse the impact of multiuser interference, as well as the effect of multipath propagation channels, on the ranging capabilities of UWB systems.

Future work will be devoted to extend the bound to more interfering pulses by including them in the system model. The performance on multipath and multiuser channels will be evaluated.

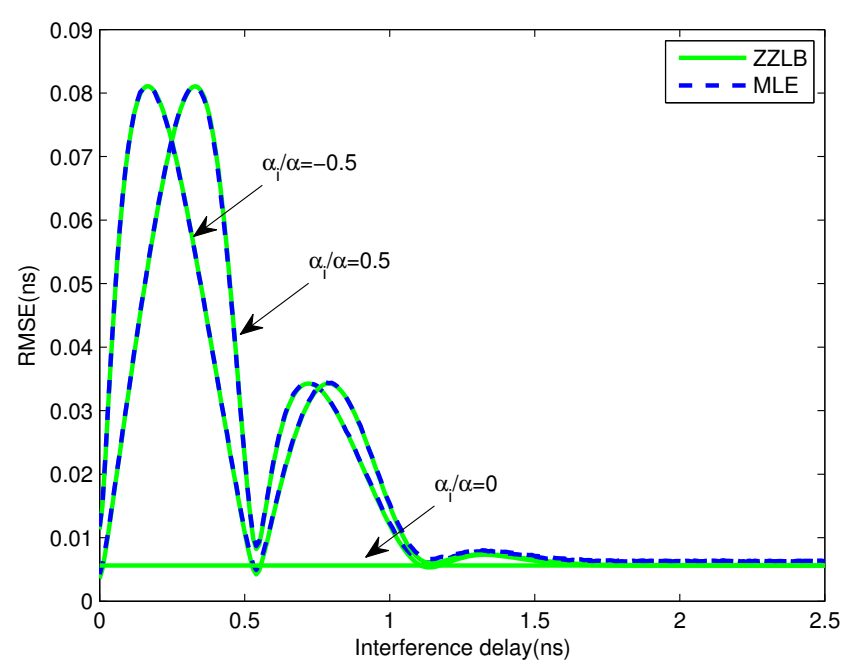

Fig. 1. RMSE with respect to $\tau_{i}$ with $\mathrm{SNR}=30 \mathrm{~dB}$.

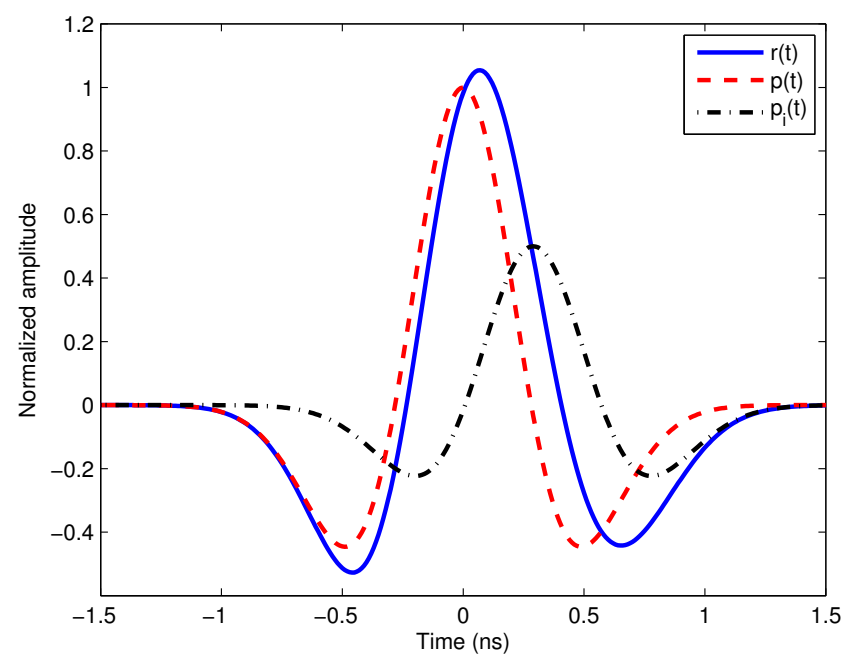

Fig. 2. Received signal when $\tau_{i}=0.3$ ns.

\section{REFERENCES}

[1] D. Dardari, A. Conti, U. Ferner, A. Giorgetti, and M. Z. Win, "Ranging With Ultrawide Bandwidth Signals in Multipath Environments," Proceedings of the IEEE, vol. 97, no. 2, pp. 404426, 2009.

[2] H. Soganci, S. Gezici, and H. V. Poor, "Accurate positioning in ultra-wideband systems," IEEE Wireless Communications Magazine, vol. 18, no. 2, pp. 19-27, 2011.

[3] J. Zhang, R.A. Kennedy, and T.D. Abhayapala, "Cramer-Rao lower bounds for the time delay estimation of UWB signals," in Communications, 2004 IEEE International Conference on, 2004, vol. 6, pp. 3424-3428.

[4] A. Mallat, C. Oestges, and L. Vandendorpe, "CRBs for UWB Multipath Channel Estimation: Impact of the Overlapping Be- 
tween the MPCs on MPC Gain and TOA Estimation,' in Proc. IEEE Int. Conf. Communications ICC '09, 2009, pp. 1-6.

[5] H. L Van Trees, Detection, Estimation, and Modulation Theory: Part I, 2nd ed, New York: John Wiley \& Sons, Inc., 2nd ed edition, 2001.

[6] D. Chazan, M. Zakai, and J. Ziv, "Improved Lower Bounds on Signal Parameter Estimation," IEEE Transactions on Information Theory, vol. 21, no. 1, pp. 90-93, 1975.

[7] K.L. Bell, Y. Steinberg, Y. Ephraim, and H.L. Van Trees, "Extended Ziv-Zakai lower bound for vector parameter estimation," Information Theory, IEEE Transactions on, vol. 43, no. 2, pp. $624-637$, mar 1997.

[8] D. Dardari, C.-C. Chong, and M. Z. Win, "Improved Lower Bounds on Time-of-Arrival Estimation Error in Realistic UWB Channels," in Ultra-Wideband, The 2006 IEEE 2006 International Conference on, 2006, pp. 531-537.

[9] Zhengyuan Xu and B. M. Sadler, "Time Delay Estimation Bounds in Convolutive Random Channels," IEEE Journal of Selected Topics in Signal Processing, vol. 1, no. 3, pp. 418430, 2007.

[10] B. M. Sadler, Ning Liu, and Zhengyuan Xu, "Ziv-Zakai Bounds on Time Delay Estimation in Unknown Convolutive Random Channels," IEEE Transactions on Signal Processing, vol. 58, no. 5, pp. 2729-2745, 2010.

[11] A. Gusi Amigo and L. Vandendorpe, "Ziv-Zakai Lower Bound for UWB based TOA Estimation with Multiuser Interference," in Proceedings of IEEE International Conference on Acoustics, Speech and Signal Processing ICASSP 2013, 2013. 